\title{
Active rheumatoid arthritis with multiple pulmonary nodules failure to multiple remissive therapy: Which is the solution?
}

\author{
Ileana Cosmina Filipescu', Milena Man' ${ }^{2}$, Simona Rednic ${ }^{1}$ \\ ${ }^{1}$ Rheumatology Department, "Iuliu Hatieganu“ University of Medicine and Pharmacy, Cluj-Napoca, Romania \\ ${ }^{2}$ Pneumology Department, "Iuliu Hatieganu" University of Medicine and Pharmacy, Cluj-Napoca, Romania
}

\begin{abstract}
We describe the case of a 63-year-old nonsmoker woman, with a long history of active seropositive rheumatoid arthritis, failure to multiple disease-modifying antirheumatic drugs due to both loss of efficacy and adverse drug reaction. She was exposed to silicon dust some years ago and has many pulmonary nodules, revealed by imaging studies as multiple cavitary lung nodules. Her initial pathological samples were negative for any infections and treatment against tuberculosis and anti-fungal therapy did not improve the appearance of the nodules. After an extensive reevaluation of pulmonary nodules, the Baricitinib treatment was started.
\end{abstract}

Keywords: rheumatoid arthritis, pulmonary nodules, synthetic DMARDs, JAK inhibitors

\section{INTRODUCTION}

Pulmonary nodules are a rare complication of rheumatoid arthritis (RA) and can be induced by many other causes like: infections, malignancies, professional exposures, drugs (1).

Tuberculosis (TB) is still a worldwide spread infectious disease caused by Mycobacterium tuberculosis (Mtb). In 2018, World Health Organization (WHO) estimated 10 million new TB cases worldwide that led to 1.5 million deaths, thus ranking TB as the main cause of mortality from a single pathogen (2). Several factors have been described as capable of considerably increasing the risk of active TB reactivation. Among them, activity of the autoimmune disorders, diabetes, corticotherapy and treatment with biological agents (3-5). The IRs (95\% $\mathrm{CI})$ of $\mathrm{TB}$ in the five large registries ranged from 0.02 $(0.01,0.03)$ to $0.35(0.17,0.67)(6,7)$.

An extensive screening to exclude infections, malignancies or other severe concomitant disorders before starting immunosuppressive treatment is mandatory in patients with RA and pulmonary nodules.

\section{CASE PRESENTATION}

We report a case of a 63-year-old nonsmoker woman affected by seropositive rheumatoid arthritis (RA) for many years (since 2004) with a long history of DMARDs failure and multiple pulmonary nodules, finally started with Olumiant. It should be noted that the patient was exposed to silica particles some years ago. Her clinical history begun when she was 43years old with arthritis of the hands, wrists, ankle and knees. On examination, there was swelling and tenderness of wrists and of all metacarpophalangeal joints and proximal interphalangeal joints, bilateral ankles and left knee (figures 1A, 1B, 1C, 1D). She also had extra-articular clinical involvement (rheumatoid nodules localized on the metacarpophalangeal II on the right hand and around the right radiocarpal joint).

The inflammatory markers C-reactive protein (CRP) and erythrocyte sedimentation rate (ESR) were $12.5 \mathrm{mg} / \mathrm{dl}$ respectively $65 \mathrm{~mm} / \mathrm{h}$ and her Disease Activity Score-28 (DAS28-ESR) at the onset was 6.26. The patient's rheumatoid factor was $256 \mathrm{U} / \mathrm{ml}$, and anti-CCP was $>200 \mathrm{U} / \mathrm{ml}$. 


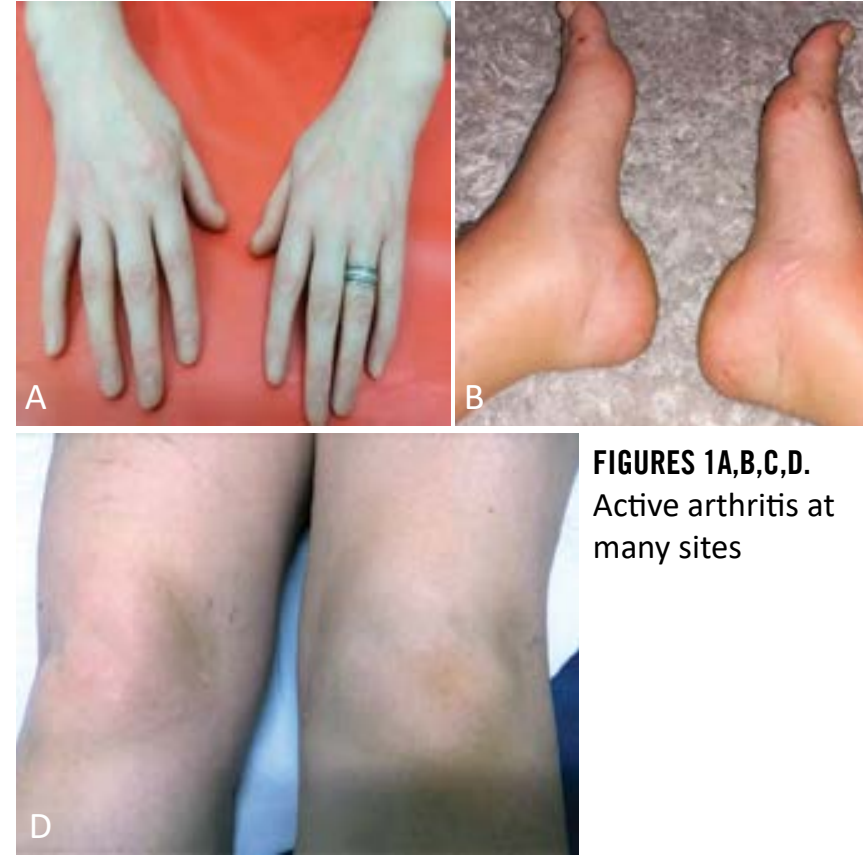

The patient was treated with methotrexate (MTX) as monotherapy up to $20 \mathrm{mg} /$ week between July 2004 - April 2010, but this treatment induced nausea, vomiting, weight loss and it was stopped. Sulfasalazine (SSZ) was started after MTX, but after almost one year was stopped also due to the reappearance of digestive symptoms. SSZ was switched with Leflunomide (20 mg daily), but after two years, in April 2018 this medications was also stopped, patient presented with fever $\left(38^{\circ} \mathrm{C}\right)$, respiratory symptoms, fatigue. Pulmonary investigations (radiography and computerized tomography scan - CT) revealed multiple pulmonary nodules on the inferior lobes, on both sides, with small dimensions, up to $26 \mathrm{~mm}$, some with central excavation. Quantiferon test was negative. Pneumologist performed a sputum test and a bronchoalveolar lavage (other infections and cancer was excluded). One subpleural nodule was removed laparoscopically. Ziehl Neelsen staining and mycobacterial culture were negative. Histopathological result confirmed lung structure with some central caseum surrounded by gigantic cells. The treatment against TB infections was started H300R600Z1500E1600 (7/7). Lefluno-

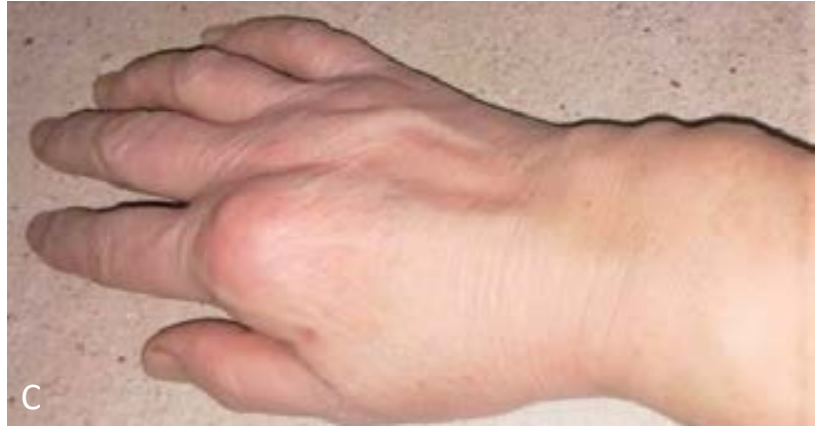

mide was stopped and replaced with Hidroxicloroquine $400 \mathrm{mg}$ daily.

She was referred to our Rheumatology Clinic in Cluj-Napoca in September 2018. The patient accused pain at many sites, difficulty walking and activities of daily living were very difficult to perform. At that time the inflammatory syndrome was severe (CRP $24 \mathrm{mg} / \mathrm{dl}$ and ESR $88 \mathrm{~mm} / \mathrm{h}$ ) and DAS28- ESR was 7.26. She had normal full blood count and basic metabolic panel levels. $\mathrm{C}$ and $\mathrm{P}$ antineutrophil cytoplasmic antibodies (ANCA) were negative. Oncologic screening was negative. Unfortunately, during Hydroxicloroquine treatment, she reported systemic reaction (urticaria) and stopped this medication. We've started her on $150 \mathrm{mg}$ Ciclosporin and $8 \mathrm{mg}$ Methylprednisolone daily.

A CT scan reevaluation done after 6 months revealed enlargement of lung nodules, without any significant change (figures 2A, 2B, 2C). Also the histopathological exam of the removed nodule was reevaluated and this time there were nor caseum nor gigantic cells reported, only incomplete necrosis with neutrophils and cellular debris without vasculitis lesions or lesions specific to silica nodules.

Ziehl Neelsen staining and mycobacterial culture were negative. Sputum tests for acid-fast bacillus (AFB) were negative thrice, and bronchoalveolar lavage fluid revealed few scattered polymorphs with no malignant cells and fungal stains and cultures for AFB were negative. IgA and IgE for Aspergillus was normal. Quantiferon test remained negative. After 9 months the specific treatment against TB was stopped. Pneumologist concluded that were
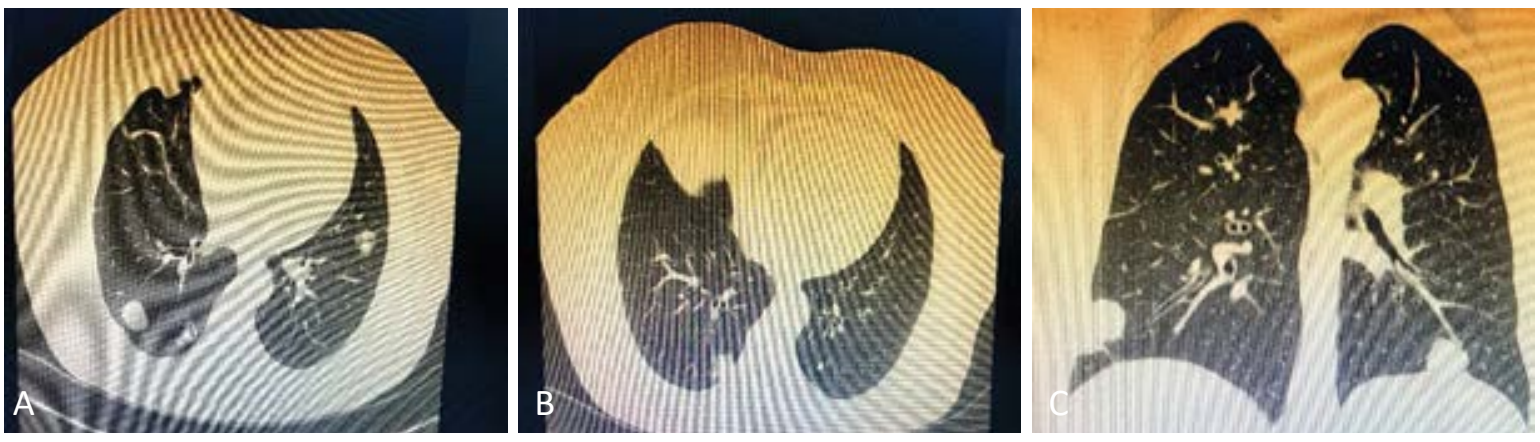

FIGURES 2A,B,C. Thoracic CT showing some aspects of nodules after specific tuberculostatic treatment 
some rheumatoid nodules that have not been influenced by anti-TB therapy and approved to start treatment with JAK inhibitor.

The DAS28-ESR score at that time was 7.22 and suggested an active arthritis. In April 2019 the patient began treatment with Baricitinib (Olumiant ${ }^{\circledR}$ ) $4 \mathrm{mg}$ daily.

The treatment was well tolerated without any adverse events. In time it decreased the use of pain relief therapy and AINS. After tree months the DAS28-ESR decreased at 3.76, with resolution of inflammatory syndrome (CRP and ESR were $7.5 \mathrm{mg} / \mathrm{dl}$ respectively $29 \mathrm{~mm} /$ ). The symptoms's resolution were so important that the patient asked to reduce the DMARD's treatment, in consequence cyclosporine was stopped and Olumiant remained as monotherapy.

Recently, in July 2021, at the last reevaluation, after 26 months of treatment with Olumiant, DAS28-ESR was 2.88. Patient accused some mechanical pain at tibiotarsal joints without any other significant problems (figures 3A, 3B).

\section{DISCUSSIONS}

The differential diagnosis of cavitary pulmonary nodules in patients with RA includes infections (fungal and mycobacterial, septic emboli), malignancies (primary or metastatic), vasculitis, drug reactions and rheumatoid nodules (1).

In our case ANCA levels were negative and there were no features of vasculitis.

Chronic silicosis typically develops slowly, usually presenting 10 to 30 years after first exposure. Chronic simple silicosis is radiologically characterized by pulmonary nodules up to $10 \mathrm{~mm}$ diameter, typically in the upper zone (7). Silica is associated with several autoimmune diseases such as systemic sclerosis (SSc), RA, systemic lupus erythematosus and antineutrophil cytoplasmic antibody (ANCA)related vasculitis, but the correlation between the level of workplace exposure and development of these disorders are unclear (potentially triggering extrapulmonary disease). In the presented case most of the lung nodules were identified in inferior zone, and the histopathogical pattern was not typical.

The prevalence of pulmonary rheumatoid nodules was $0.4-32 \%$ depending on the imagistic tools (8). Frequently occuring in patients with longstanding disease, the nodules and are typically located along the interlobular septa or in subpleural regions, usually in men (7:1 ratio) (1). They are rarely symptomatic, although they can present with cough and hemoptysis. Usually, these regress with standard DMARD therapy but paradoxically has been shown to be accelerated by methotrexate, induced

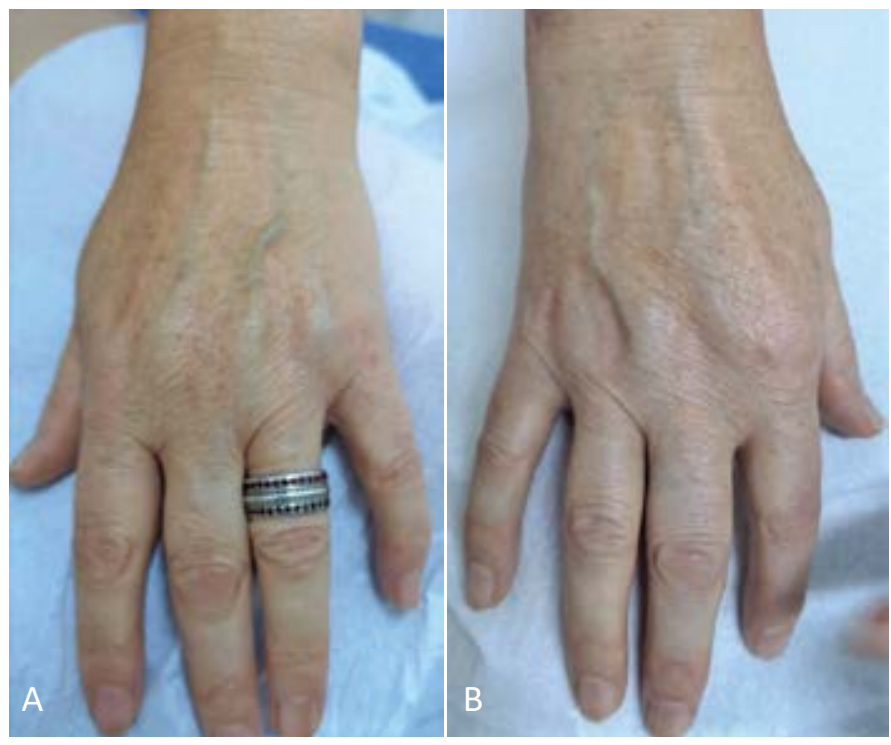

FIGURES 3A,B. Hands without arthritis after 26 months with Olumiant treatment

by leflunomide, azathioprine, and antitumor necrosis factor (anti-TNF) etanercept, and infliximab (1,911). In some cases can be colonized with fungus (1).

Reactivation of TB involves the upper lobes, while primary TB usually occurs as a lower lobe disease. The use of TNF inhibitors increased the risk of $\mathrm{TB}$, with hazard ratios of 2.7-12.5, compared with that in the general population especially in an endemic area of the disease, and the adjusted hazard ratio $(95 \% \mathrm{CI})$ versus csDMARDs was between 2.7 $(2.1,3.3)$ and $4.9(2.1,11.1)$ after $2013(5,12,13)$. Extrapulmonary tuberculosis was more common in patients treated with TNF inhibitors but was also observed in tofacitinib and baricitinib treated patients (14-16).

A very recently published systematic review conducted by Cantini et al assess the frequency of active TB occurrence in patients with RA treated with tofacitinib, baricitinib, upadacitinib, and filgotinib through a systematic review of available RCTs, open label extension (OLE) of RCTs, National databases and registries. In all 40 reports $1,79(0.28 \%)$ active TB cases was found in 28,099 tofacitinib-exposed patients, $10(0.23 \%)$ in 4310 patients treated with baricitinib, while no cases were observed in patients treated with other two JAK inhibitors. None of the $10 \mathrm{~TB}$ cases observed in baricitinib trials were recorded in low TB endemic areas and 79 TB cases in tofacitinib-exposed were observed in high or intermediate TB risk countries (16).

In 2019, Masayoshi Harigai found 26 cases of TB in the tofacitinib-treated patients of phase III and LTE studies after 64 weeks (range 15-161). Fifteen (58\%) cases involved extrapulmonary infection. He reported that also two TB cases were during the placebo-controlled period (e.g., 0 to week 24): one in the baricitinib 4-mg group $(\mathrm{n}=997)$ and one in the 
adalimumab group $(n=330)$. Six TB cases were reported during the uncontrolled period, and the bacteria was not identified in three of them. These TB cases were reported from endemic areas of the disease (12).

A comparison of the IRs of TB between JAK inhibitor- and TNF inhibitor-treated patients in clinical settings remains to be established in each country. A screening tools, prevention and monitoring latent TB infection is similar between JAK inhibitors and biological DMARDs (17-19).

In our case the inferior part of the lung was especially affected by nodules, the extensive TB evaluations were negative and the specific medications against TB didn't improve the aspects of the pulmonary structures. The low incidence of active TB re-

Conflict of interest: none declared

Financial support: none declared corded for JAK inhibitors increases the confidence in this therapeutic choice when treating patients with RA and with some risk for TB.

\section{CONCLUSIONS}

The evolution of rheumatoid lung nodules during the disease is not predictable. Many other causes can induce pulmonary nodules and it is very important to exclude severe disorders. Some of the immunosuppression drugs can induce nodules. JAK inhibitors are effective and safe in patient with active RA and risk to develop TB. After more than 2 years of monotherapy with Olumiant our patient is feeling well, is in a low disease activity, without any significant adverse events or respiratory symptoms.

\section{REFERENCES}

1. Wickrematilake G. Complicated Rheumatoid Nodules in Lung. Case Reports in Rheumatology. 2020;2020:6627244.

2. WHO. Global tuberculosis report. 2019. Available at: https:// www.who.int/tb/publications/global_report/en/.

3. Minozzi $\mathrm{S}$, Bonovas $\mathrm{S}$, Lytras $\mathrm{T}$, et al. Risk of infections using anti-TNF agents in rheumatoid arthritis, psoriatic arthritis, and ankylosing spondylitis: a systematic review and meta-analysis. Expert Opin Drug Saf. 2016:15:11-34.

4. Cantini F, Niccoli L, Capone A, et al. Risk of tuberculosis reactivation associated with traditional disease modifying antirheumatic drugs and non-anti-tumor necrosis factor biologics in patients with rheuamatic disorders and suggestion for clinical practice. Expert Opin Drug Saf. 2019;18(5):415-425.

5. Sepriano A, Kerschbaumer A, Smolen JS, van der Heijde D, et al. Safety of synthetic and biological DMARDs: a systematic literature review informing the 2019 update of the EULAR recommendations for the management of rheumatoid arthritis. Ann Rheum Dis. 2020 Jun;79(6):760-770.

6. Yamanaka $\mathrm{H}$, Askling J, Berglind N, Franzen S, Frisell T, et al. Infection rates in patients from five rheumatoid arthritis (RA) registries: contextualising an RA clinical trial programme. $R M D$ Open. 2017 Oct 10;3(2):e000498.

7. Hoy RF, Chambers DC. Silica-related diseases in the modern world. Allergy. 2020 Nov;75(11):2805-2817.

8. Sagdeo P, Gattimallanahali Y, Kakade G, Canchi B. Rheumatoid lung nodule. BMJ Case Rep. 2015 Oct 29;2015:bcr2015213083.

9. Akiyama N, Toyoshima M, Kono M, Nakamura Y, Funai K, Suda T. Methotrexate-induced Accelerated Pulmonary Nodulosis. Am J Respir Crit Care Med. 2015 Jul 15;192(2):252-3.

10. Yoshikawa GT, Dias GA, Fujihara S, Silva LF, Cruz Lde B, Fuzii HT, Koyama RV. Formation of multiple pulmonary nodules during treatment with leflunomide. J Bras Pneumol. 2015 MayJun;41(3):281-4.

11. Martín-de León R, Moisés J, Peris P, Agustí C, Marrades RM. Nódulos pulmonares cavitados en paciente tratada con anti-

TNF. Arch Bronconeumol. 2018;54:431-432.

12. Harigai M. Growing evidence of the safety of JAK inhibitors in patients with rheumatoid arthritis. Rheumatology (Oxford). 2019 Feb 1;58(Suppl 1):i34-i42.

13. Ramiro S, Sepriano A, Chatzidionysiou K, Nam JL, Smolen JS, van der Heijde $D$, et al. Safety of synthetic and biological DMARDs: a systematic literature review informing the 2016 update of the EULAR recommendations for management of rheumatoid arthritis. Ann Rheum Dis. 2017 Jun;76(6):1101-1136.

14. Winthrop KL, Novosad SA, Baddley JW, Calabrese L, et al. Opportunistic infections and biologic therapies in immunemediated inflammatory diseases: consensus recommendations for infection reporting during clinical trials and postmarketing surveillance. Ann Rheum Dis. 2015 Dec;74(12):2107-16.

15. Winthrop KL, Lindsey S, Harigai M, et al. Potential opportunistic infections, and other infections of interest in patients with moderate to severe rheumatoid arthritis in the baricitinib program (Abstract 2787). 2017 ACR/ARHP Annual Meeting. Arthritis Rheumatol. 2017;69:Abstract 2787.

16. Cantini F, Blandizzi C, Niccoli L, Petrone L, Goletti D. Systematic review on tuberculosis risk in patients with rheumatoid arthritis receiving inhibitors of Janus Kinases. Expert Opin Drug Saf. 2020 Jul;19(7):861-872.

17. Atzeni F, Talotta R, Nucera V, et al. Adverse events, clinical considerations and management recommendations in rheumatoid arthritis patients treated with JAK inhibitors. Expert Rev Clin Immunol. 2018;14(11):945-956.

18. Yeo-Jin S, Soo-Kyung C, Hyoungyoung K, et al. Risk of Tuberculosis Development in Patients with Rheumatoid Arthritis Receiving Targeted Therapy: a Prospective Single Center Cohort Study. J Korean Med Sci. 2021 Mar 15;36(10):e70.

19. Nash P, Lim I, Marabani M. A comparison of Janus kinase inhibitor safety in rheumatoid arthritis. International Journal of Rheumatic Diseases. 2021:24(S1): 3-14. 